\title{
Interplay between Strong Coupling and Radiative Damping of Excitons and Surface Plasmon Polaritons in Hybrid Nanostructures
}

\author{
Wei Wang, ${ }^{\dagger}$ Parinda Vasa, ${ }^{t, \neq}$ Robert Pomraenke, ${ }^{\dagger}$ Ralf Vogelgesang, ${ }^{\dagger}$ Antonietta De Sio, ${ }^{\dagger}$ Ephraim Sommer, ${ }^{\dagger}$ \\ Margherita Maiuri, ${ }^{\S}$ Cristian Manzoni, ${ }^{\S}$ Giulio Cerullo, ${ }^{\S}$ and Christoph Lienau ${ }^{\dagger, *}$ \\ ${ }^{\dagger}$ Institut für Physik and Center of Interface Science, Carl von Ossietzky Universität Oldenburg, D-26111 Oldenburg, Germany, ${ }^{\ddagger}$ Department of Physics, Indian Institute

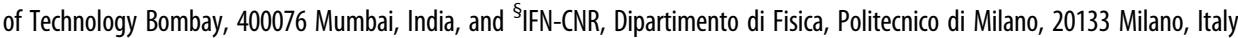

\begin{abstract}
We report on the interplay between strong coupling and radiative damping of strongly coupled excitons (Xs) and surface plasmon polaritons (SPPs) in a hybrid system made of J-aggregates and metal nanostructures. The optical response of the system is probed at the field level by angle-resolved spectral interferometry. We show that two different energy transfer channels coexist: coherent resonant dipole-dipole interaction and an incoherent exchange due to the spontaneous emissions of a photon by one emitter and its subsequent reabsorption by another. The interplay between both pathways results in a pronounced modification of the radiative damping due to the formation of super- and subradiant polariton states. This is confirmed by probing the ultrafast nonlinear response of the polariton system and
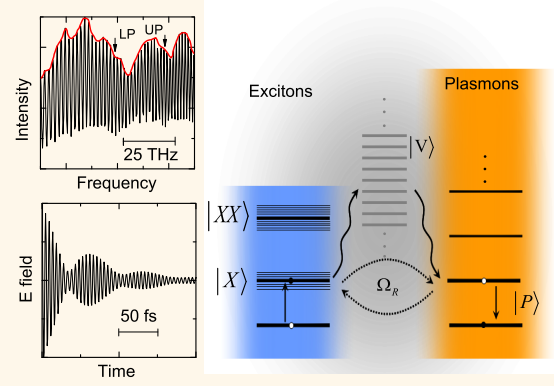
explained within a coupled oscillator model. Such a strong modification of the radiative damping opens up interesting directions in coherent active plasmonics.
\end{abstract}

KEYWORDS: exciton-plasmon coupling $\cdot$ molecular aggregates $\cdot$ metallic nanostructures $\cdot$ sub- and superradiance $\cdot$ spectral interferometry $\cdot$ ultrafast coherent spectroscopy

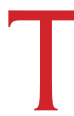
wo quantum emitters, placed in close proximity, can radiatively couple not only to the environment, but also to each other. ${ }^{1,2}$ If the coupling strength between the two individual systems exceeds the damping of either, the strong coupling regime is reached. In this case, two distinct interactions can contribute to the radiative coupling: ${ }^{3,4}$ (i) a coherent exchange of photon energy between the two emitters due to their resonant dipole-dipole interaction, ${ }^{2}$ preserving the relative phase between their optical polarizations. This exchange leads to the formation of hybridized states. $^{1,5,6}$ (ii) One emitter can spontaneously emit a photon which is then reabsorbed by the other emitter, resulting in an incoherent exchange of photon energy. Both interactions are mediated by vacuum field fluctuations and exist even in the absence of any external excitations. ${ }^{7,8}$ The interplay between both coherent and incoherent interaction processes not only changes the energetics of the system. It also governs its dynamics by altering the radiative damping of these modes, resulting in cooperative emission phenomena known as sub- and superradiance. ${ }^{9}$ These are of great interest as they play a dominant role in the optical properties of many strongly coupled systems such as trapped ions, ${ }^{1}$ molecular aggregates, ${ }^{10}$ excitonic quantum dots $^{11}$ and wells, ${ }^{12}$ and plasmonic excitations in nanostructures. ${ }^{13-17}$

Recently, strong coupling between fundamentally distinct emitters has been studied extensively, ${ }^{17}$ e.g., in hybrids comprised of excitons (Xs) in organic/inorganic semiconductors or J-aggregated molecules and plasmonic nanostructures supporting surface plasmon polaritons (SPPs). ${ }^{5,18-24}$ In these systems, vacuum field fluctuations lead to a coherent exchange of energy between ensembles of excitons and plasmon modes and the formation of hybridized X-SPP states. This has attracted
* Address correspondence to christoph.lienau@uni-oldenburg.de.

Received for review November 19, 2013 and accepted December 30, 2013.

Published online

10.1021/nn405981k

(c) XXXX American Chemical Society 
considerable interest due to possible applications in plasmonic switching ${ }^{25,26}$ and lasing. ${ }^{27}$ Although the coherent interaction and the corresponding stationary properties have been studied in detail, and Rabi splittings as large as several hundreds of millielectronvolts have been demonstrated, ${ }^{5,18,21,23}$ so far little is known about the incoherent interaction channel and thus about the effects of strong coupling on the radiative damping of such hybrid systems.

Here, we focus on hybrid nanostructures comprised of J-aggregate excitons and surface plasmon polaritons, a prototype system featuring strong coupling between fundamentally distinct excitations. $5,18,21,23$ We explore their radiative properties in angle- and phase-resolved linear optical spectroscopy and find evidence for the formation of subradiant and superradiant X-SPP hybrid states. In particular, when bringing excitons and SPPs into resonance, we observe distinctly different spectral linewidths for these two hybrid modes. This indicates that in our system a vacuum-mediated incoherent dipole coupling leads to different radiative lifetimes of the two polariton modes. This is confirmed by time-resolved measurements of ultrashort polariton lifetimes, giving clear evidence for sub- and superradiance in hybrid plasmonic nanostructures.

\section{RESULTS AND DISCUSSION}

Incoherent Dipole Coupling between Two Strongly Coupled Modes. Here, we seek to briefly illustrate the general effects of coherent and incoherent dipolar couplings on the optical and electronic properties of a strongly coupled hybrid system. For this, we first analyze these effects for a simple model system of a single exciton coupled to a photon-like SPP oscillator. ${ }^{3,28-31}$ We describe the optical resonances of the uncoupled $X$ and SPP-systems by their complex resonance frequencies $\widetilde{\omega}_{\mathrm{X}}=\omega_{\mathrm{X}}-i \gamma_{\mathrm{X}}$ and $\widetilde{\omega}_{\mathrm{P}}=\omega_{\mathrm{P}}-i \gamma_{\mathrm{P}}$, respectively. Their real parts give the eigenenergies $\hbar \omega_{\mathrm{X}}$ and $\hbar \omega_{\mathrm{P}}$, and the imaginary parts $\gamma_{\mathrm{X}}=\Gamma_{\mathrm{X}} / 2=1 / 2 T_{1, \mathrm{X}}$ and $\gamma_{\mathrm{P}}=$ $\Gamma_{\mathrm{P}} / 2=1 / 2 T_{1, \mathrm{p}}$, respectively, denote population damping by radiative and/or nonradiative processes. Here, the $X$ and SPP population lifetimes are $T_{1, X}$ and $T_{1, P}$, respectively.

We assume that both systems are interacting via a dipolar (Rabi) coupling between the excitonic transition dipole moment and the SPP field. The Rabi coupling is described by a Hermitian matrix element $\Omega_{\mathrm{R}}$. We are interested in the strong coupling limit. In the present model, it is reached when $\Omega_{\mathrm{R}}$ exceeds $\left(\gamma_{\mathrm{X}}+\gamma_{\mathrm{P}}\right) / 2 .{ }^{29,32}$ In this limit, the Rabi oscillations become noticeable in the time domain and two separate resonances emerge in the frequency domain, i.e., in the linear optical spectra. If both systems are brought into resonance, the coupling can result in a coherent exchange of energy between them, oscillating with a Rabi period of about $\pi /\left|\Omega_{R}\right|$ (dashed arrows

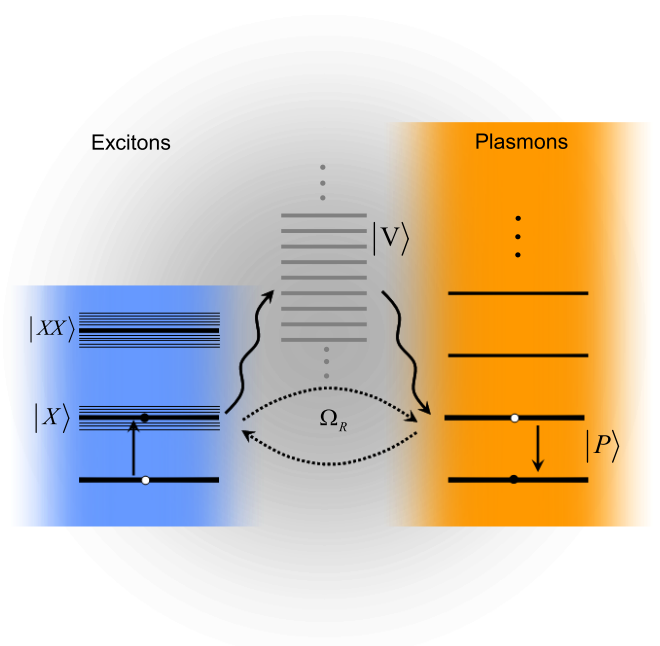

Figure 1. Schematic of a strongly coupled $X$-SPP system. The excitonic system is modeled as a three-level system consisting of a ground state, a single exciton state $|X\rangle$ and a biexciton state $|X X\rangle$. The plasmon system is represented as a photonic mode $|P\rangle$. The continuum of vacuum states is denoted as $|V\rangle$. The dashed arrows represent the coherent Rabi coupling $\Omega_{R}$ and the solid arrows denote the incoherent $X-S P P$ coupling through vacuum field.

in Figure 1). ${ }^{30}$ Also, both X and SPP can spontaneously emit photons into the surrounding vacuum field modes. These photons can then be reabsorbed, resulting in an incoherent exchange of energy between both systems, without any definite phase relationship. This process can be described by a non-Hermitian matrix element $\hbar \gamma_{\text {XP, }}$, the so-called cross-damping term. ${ }^{3,33,34}$ It is important that $\gamma_{\mathrm{XP}}$ and $\Omega_{\mathrm{R}}$ are not independent of each other but are related by a Kramers-Kronig relationship. ${ }^{3}$ We will show later that both channels coexist and play an important role for the radiative damping of our hybrid system.

For describing the linear optical response of the coupled system, only the lowest single-exciton and single-plasmon excitations of the system are relevant. We can then describe the system by an effective nonHermitian Hamiltionian in the form of a $2 \times 2$ matrix $^{13,30}$

$$
\hat{H}=\hbar\left[\left(\begin{array}{ll}
\widetilde{\omega}_{\mathrm{X}} & \Omega_{\mathrm{R}} \\
\Omega_{\mathrm{R}} & \widetilde{\omega}_{\mathrm{P}}
\end{array}\right)-i\left(\begin{array}{ll}
0 & \gamma_{\mathrm{XP}} \\
\gamma_{\mathrm{XP}} & 0
\end{array}\right)\right]
$$

By diagonalizing this coupling matrix, the hybrid superposition modes of the coupled system, i.e., the (energetically) lower (LP) and upper (UP) polariton mode, are found. Their dispersions, $\mathscr{R}\left(\widetilde{\omega}_{ \pm}\right)$, are given by the real parts and the damping rates, $\Gamma_{ \pm}=2 \gamma_{ \pm}=2\left|\mathcal{2}\left(\widetilde{\omega}_{ \pm}\right)\right|$, by the imaginary parts of the hybrid mode energies

$$
\begin{aligned}
& \hbar \tilde{\omega}_{ \pm}=\hbar\left(\frac{\tilde{\omega}_{\mathrm{X}}+\tilde{\omega}_{\mathrm{P}}}{2}\right) \\
& \pm \hbar \sqrt{\left(\frac{\widetilde{\omega}_{\mathrm{X}}-\tilde{\omega}_{\mathrm{P}}}{2}\right)^{2}+\left(\left|\Omega_{\mathrm{R}}\right|^{2}-\gamma_{\mathrm{XP}}^{2}\right)-2 i \gamma_{\mathrm{XP}} R\left(\Omega_{\mathrm{R}}\right)}
\end{aligned}
$$


More details are provided in the Materials and Methods section.

To illustrate the critical importance of the crossdamping term, we discuss here the two extreme cases of (i) two identical oscillators, and (ii) two nonidentical emitters with distinctly different radiative lifetimes. Equation 2 shows that, in the strong coupling regime and at zero detuning $\left(\mathscr{R} \widetilde{\omega}_{\mathrm{x}}=\mathscr{R} \widetilde{\omega}_{\mathrm{p}}\right)$, a difference in the damping rates of the hybrid modes is found only if the last term under the root does not vanish, i.e., the incoherent coupling coefficient $\gamma_{\mathrm{XP}}=\Gamma_{\mathrm{XP}} / 2$ must be nonzero. Figure $2(a, b)$ illustrates case $(i)$, in which both emitters have identical damping rates $\Gamma_{\mathrm{X}}=2\left|\mathcal{L}\left(\widetilde{\omega}_{\mathrm{X}}\right)\right|=$ $\Gamma_{\mathrm{P}}=2\left|\mathcal{L}\left(\widetilde{\omega}_{\mathrm{P}}\right)\right|=\Gamma_{0}$. If the incoherent cross-damping term is ignored, the coupled system behaves like a classical coupled-oscillator system with the same decay rate $\left(\Gamma_{0}\right)$ in both hybrid modes (dashed line in Figure 2a), independent of the mixing ratio $\left|C_{X}\right|^{2}$ and $\left|C_{P}\right|^{2}$ giving the probability of finding the system in either the exciton or plasmon state (Figure $2 b$ ). If $\Gamma_{X P}=$ $\left(\Gamma_{\mathrm{X}} \cdot \Gamma_{\mathrm{P}}\right)^{1 / 2}$ is taken into account, ${ }^{3}$ however, bright superradiant (solid blue line) and dark subradiant (solid red line) modes are formed. On resonance, the former decays at $2 \Gamma_{0}$, i.e., twice the individual rate, whereas the latter does not decay at all, since this superposition is effectively decoupled from the photonic bath.

More appropriate for the actual sample system discussed in the present report is case (ii). We assume a fast decay rate of $\Gamma_{\mathrm{P}}=50 \mathrm{ps}^{-1}$ and a distinctly slower decay rate $\Gamma_{X}=1 \mathrm{ps}^{-1}$, independent of detuning. As shown in Figure $2 c$ (dashed lines), the decay rates of the coupled modes mainly represent the mixing ratios
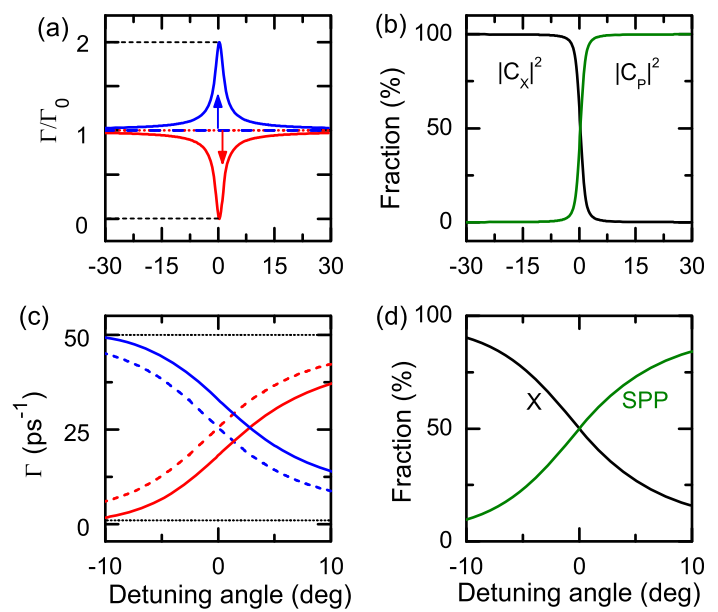

Figure 2. (a and c) Radiative damping rates of hybrid modes in the presence (solid lines) and absence (dashed lines) of cross damping for the case of two identical coupled dipole emitters (a) and two coupled dipole emitters with distinctly different radiative decay rates (c). Here, a blue line denotes the superradiant and a red line the subradiant mode. (b and d) Mixing fractions for the subradiant hybrid state in the case of two identical coupled dipole emitters (b) and two coupled dipole emitters with distinctly different radiative decay rates $(\mathrm{d})$.

of the $X$ and SPP (Figure 2d), if the incoherent crossdamping is neglected. On resonance, this results in identical decay rates $\left(\Gamma_{\mathrm{X}}+\Gamma_{\mathrm{P}}\right) / 2$ for both hybrid modes. This changes in the presence of cross-damping (taking $\Gamma_{\mathrm{XP}}=\left(\Gamma_{\mathrm{X}} \cdot \Gamma_{\mathrm{P}}\right)^{1 / 2}$ ), with a clear nondegeneracy of the decay rates at zero detuning (solid lines). We see that, on resonance, hybrid modes with an equal admixture of X and SPP wave functions, yet with distinctly different radiative decay rates, are formed. Both cases thus illustrate that this formation of short-lived (superradiant) and long-lived (subradiant) hybrid states with distinctly different decay rates at zero detuning is the main signature of an incoherent cross-damping.

Linear Optical Response of the $\mathbf{X}$-SPP Hybrid System. We study J-aggregate/metal hybrid nanostructures, composed of a 50-nm-thick J-aggregate dye film, spincoated on gold nanogroove arrays. These arrays are made of relatively shallow (30-nm deep) depressions with a width of about $50 \mathrm{~nm}$, carved into the gold surface by focused ion beam milling, as schematically shown in Figure 3a. The periods of the nanogrooves of $400-460 \mathrm{~nm}$ are properly chosen such that angletuning can continuously shift the active SPP resonances to energies above and below the exciton resonance at $\sim 1.79 \mathrm{eV}$, effectively setting the $\mathrm{X}$-SPP detuning. The shallow depth and the narrow width of the grooves ensure spectrally narrow SPP resonances with correspondingly long lifetimes. ${ }^{21,30}$ High-resolution, angleresolved spectral interferometry, ${ }^{13,35}$ as schematically depicted in Figure $3 \mathrm{~b}$, is used to characterize amplitude and phase of the linear optical response of the hybrid system, specifically the spectral lineshapes.

(a)

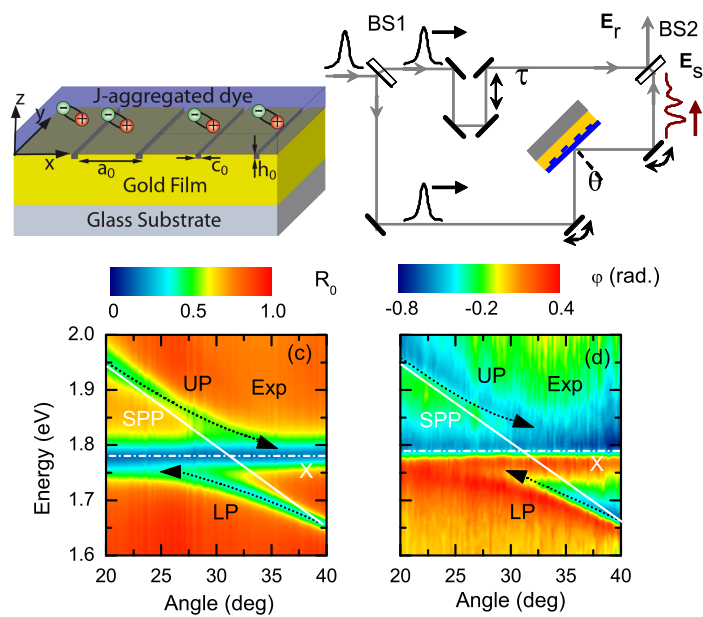

Figure 3. (a) Schematic of the hybrid structure consisting of a gold nanogroove array coated with a 50-nm-thick J-aggregate dye film. (b) Angle-resolved spectral interferometry setup (BS, beam splitter; $\tau$, variable delay). Observed angleresolved reflectivity spectra (c), $R_{0}(\theta, \omega)$ and corresponding observed spectral phase (d), $\varphi(\theta, \omega)$. The black (white) lines in (c) and (d) mark the coupled (uncoupled) mode dispersions. 
The measured angle-dependent reflectivity amplitude $\left(R_{0}(\omega)\right)$ and phase $(\varphi(\omega))$ spectra are shown in Figure 3, panels $c$ and $d$, respectively. We find the typical anticrossing behavior of the $X$ and SPP resonances, indicating a strong $X-S P P$ coupling that results in the formation of a higher (UP) and lower energy (LP) hybrid polariton mode. These spectra are recorded using low-energy pulses from an ultrafast white-light source and are independent of the laser fluence in the range from 0.1 to $100 \mathrm{~nJ} / \mathrm{cm}^{-2}$. For such low fluences, the average occupation number for the excitonic and photonic modes is much less than unity $^{21,30}$ ensuring that the $X$-SPP coupling is independent of the external laser excitation. Instead, fluctuations of the vacuum-field induce a strong dipolar coupling ${ }^{29}$ between the ensemble of J-aggregate excitons and the resonant SPP modes. This strong $X-S P P$ coupling results in a normal mode splitting ${ }^{36}$ of the UP and LP resonances of $2 \hbar \Omega_{\mathrm{R}}$. There is also a prominent absorption band at the unperturbed J-aggregate exciton resonance $(\sim 1.79 \mathrm{eV})$. Evidently, a significant portion of the uniformly distributed molecules remains uncoupled, presumably due to the sharp variation of SPP field strength in space. The main SPP field enhancement is localized to small volumes near the grooves. J-aggregated molecules located in these regions can strongly couple to the SPP fields. Excitons outside these regions may not or only weakly couple to the SPP field. ${ }^{21,30}$ Each of the three resonances is characterized by a clear jump in the spectral phase (Figure 3d).

In such nanogroove arrays, both the narrow $X$ and SPP resonance interfere with a broadband reflection from the gold interface, resulting in typical Fano-like line shapes. ${ }^{13,20,37}$ Phenomenologically, we can describe the complex reflectivity coefficient $r(\omega)$ of the coupled system by fitting a sum of oscillator response functions to $R_{0}(\omega)$ and $\varphi(\omega)$ (Figure 3c,d) according to

$$
\begin{aligned}
r(\omega) & =\sqrt{R_{0}(\omega)} \mathrm{e}^{i \varphi(\omega)} \\
& =\alpha+i \beta \sum_{m}\left[\frac{\left|\mu_{\mathrm{m}}\right|^{2} \mathrm{e}^{-i \phi_{\mathrm{m}}}}{\omega-\omega_{\mathrm{m}}+i \gamma_{\mathrm{m}}}+\frac{\left|\mu_{\mathrm{m}}\right|^{2} \mathrm{e}^{i \phi_{m}}}{\omega+\omega_{\mathrm{m}}+i \gamma_{\mathrm{m}}}\right]
\end{aligned}
$$

Here, $\alpha$ and $\beta$ are real-valued, slowly varying background and scaling amplitudes, respectively. The indices $m=1, \ldots, 4$ represent LP, UP, the uncoupled J-aggregate resonance, and an additional broad shoulder at $\sim 2 \mathrm{eV}$ attributed to higher vibronic states or some residual dye monomer. $\boldsymbol{\mu}_{\mathrm{m}}, \phi_{\mathrm{m}}$ and $\omega_{\mathrm{m}}$ denote the effective dipole moment, phase, and resonance frequency of the individual resonances, respectively. The spectral full width at half-maximum (FWHM) is characterized as $2 \gamma_{\mathrm{m}}$.

We compare the measured with the fitted results in Figure $4 a, b$ for angles of incidence of $25^{\circ}$ (large
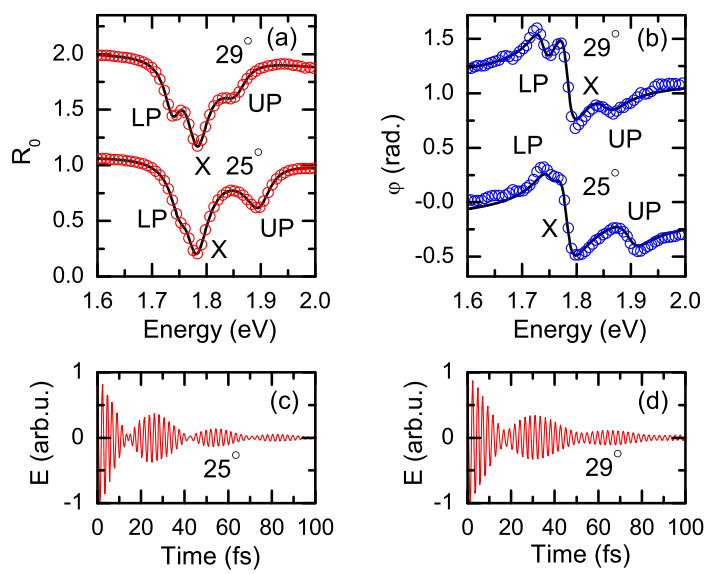

Figure 4. ( $a$ and $b$ ) Experimental (circles) and simulated (solid lines) reflectivity spectra (a) and spectral phases (b) at $\theta=25^{\circ}$ and $29^{\circ}$ (vertically shifted). (c and d) Time structure of the electric field emitted by the hybrid UP and LP modes at $25^{\circ}$ (c) and $29^{\circ}$ (d), showing distinct polarization beats with frequency $\omega_{\mathrm{UP}}-\omega_{\mathrm{LP}}$.

detuning) and $29^{\circ}$ (small detuning). In both cases, both the amplitude and phase spectra are quantitatively reproduced by the Lorentzian oscillator model eq 3 . This suggests that a possible inhomogeneous broadening of the ensemble of coupled polariton modes has only a minor influence on their optical spectra. From eq 3 , we can extract the polariton response and deduce the time structure of the re-emitted polariton field under weak impulsive excitation. As shown in Figure $4 c, d$, the field shows polarization oscillations with a beat frequency $\omega_{\mathrm{UP}}-\omega_{\mathrm{LP}}$ within the short damping time of $<100 \mathrm{fs}$. The variation of this beat frequency with angle shows a clear anticrossing, the signature of vacuum-field Rabi oscillations ${ }^{29,36,38}$ between the exciton ensemble and the resonant SPP modes in the spectral domain. Time-resolved experiments ${ }^{30}$ provide independent evidence that this beating reflects a periodic energy transfer between Xs and SPPs.

The deduced polariton characteristics are represented by circles in Figure 5: The zero detuning angle is found at $\theta_{0}=31^{\circ}$. At this angle of incidence, hybrid $X$-SPP modes are excited that contain fractions of $50 \%$ $X$ and $50 \%$ SPP. From Figure $5 a$, we find a normal mode splitting of the UP and LP branches of $2 \hbar\left|\Omega_{R}\right|=$ $114 \mathrm{meV}$ at zero detuning. Interestingly, Figure $5 \mathrm{c}$ exhibits a pronounced anticrossing of the angledependent polariton widths. Even at zero detuning, a width for the UP mode is extracted that is much broader than the average width of the individual $X$ and SPP modes, whereas that of the LP mode is significantly narrower. The UP FWHM exceeds that of the LP branch by $\sim 20 \mathrm{meV}$.

Theoretical Modeling of $\mathbf{X}$-SPP System. The observed difference in UP and LP width might be taken as an indication that incoherent cross-damping affects the radiative lifetimes and thus the linewidths of the hybrid modes. Further evidence for this assertion is provided 

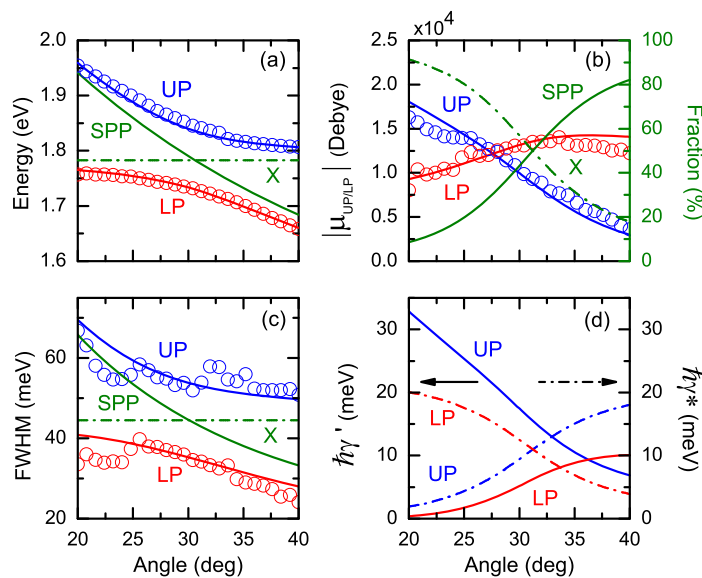

Figure 5. (a) Dispersion relations, (b) effective dipole moments and (c) spectral widths of the UP/LP modes obtained from experiment (circles) and oscillator model (solid lines). Dispersions (a) and widths (c) of the uncoupled modes are shown in green. (b) The modeled SPP- (solid) and X- (dashdot) fraction of the LP mode is shown in green. (d) Calculated population damping ( $\hbar \gamma^{\prime}$, solid) and pure dephasing ( $\hbar \gamma^{*}$, dash-dot) of the UP (blue) and LP (red).

by a coupled oscillator model, which describes the observed angle-dependent optical response successfully only if both coherent resonant dipole-dipole interaction and incoherent photon exchange processes are taken into account.

In the following, we assume the linear optical spectra of the X-SPP system are predominantly homogeneously broadened. Experimental evidence that supports this assumption will be discussed in the next section. When neglecting inhomogeneous broadening effects, the linear optical spectra of the coupled system can be modeled in terms of the effective Hamiltonian eq 1 using standard perturbation theory. ${ }^{39}$ The complex-valued eigenenergies of the polariton modes and their effective dipole moments can be directly deduced from the coupling matrix, eq 1 (see Materials and Methods). The population lifetimes of the polariton modes are given as $1 / 2 \gamma_{ \pm}^{\prime}=1 /\left(2\left|\mathscr{T}\left(\widetilde{\omega}_{ \pm}\right)\right|\right)$and contribute to the line width of the optical spectra. In addition, the total spectral widths $\gamma_{ \pm}=$ $\gamma_{ \pm}^{\prime}+\gamma_{ \pm}^{*}$ may contain contributions $\gamma_{ \pm}^{*}$ from pure dephasing. Whereas pure dephasing is negligible for the SPP system, ${ }^{40}$ it typically dominates the spectra of J-aggregated dyes ${ }^{41}$ at room temperature. We therefore expect that pure dephasing of the polariton modes scales linearly, to first order, with the exciton fraction (Figure $5 b$ ).

To compare this model with experimental data, the angle-dependent SPP dispersion relation and width was deduced from optical spectra of a PVA-coated grating without dye. ${ }^{20,21}$ With this input, the $X-S P P$ polariton dispersions shown in Figure 5 a were obtained from eq 1 for optimized values of $\hbar\left|\Omega_{R}\right|=$ $57 \mathrm{meV}$ and $\hbar \gamma_{\mathrm{XP}}=-10 \mathrm{meV}$. Angle-dependent values of $\hbar \gamma_{ \pm}^{\prime}$ and $\hbar \gamma_{ \pm}^{*}$ are displayed in Figure $5 d$ and

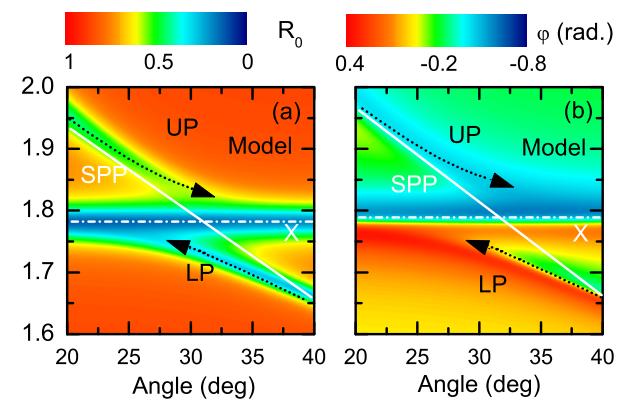

Figure 6. (a) Calculated angle-resolved spectra, $R_{0}(\theta, \omega)$ and (b) spectral phase, $\varphi(\theta, \omega)$. The black (white) lines mark the coupled (uncoupled) mode dispersions.

the resulting total polariton widths $2 \hbar \gamma_{ \pm}$are shown in Figure $5 c$. Reasonable agreement with experiment is found and in particular the observed anticrossing is reproduced, provided that a finite value for the cross damping rate $\gamma_{X P}$ is taken into account.

Using these parameters, we have simulated optical spectra from eq 3, using effective dipole moments of the UP and LP oscillators as shown in Figure $5 \mathrm{~b}$. The simulated angle-dependent amplitude and phase spectra match very well with experiment, as displayed in Figure 6. This evidence points strongly to very different UP and LP population decay rates at $\theta_{0}$ and to the presence of incoherent photon exchange between excitons and SPPs as the cause for the line width anticrossing in Figure 5c. This is an interesting result that relates the narrow LP line width to the emergence of sub- and superradiance in the coupled polariton system. Narrow LP linewidths have also been observed in earlier work on semiconductor microcavities ${ }^{42}$ and also in a study of J-aggregate molecules in metal nanovoids. ${ }^{19}$ It has been related to polariton motional narrowing ${ }^{43,44}$ or disorder effects on the Rabi coupling. ${ }^{45}$ However, an alternative explanation may be due to influences of inhomogeneity, which results in motional narrowing effects. ${ }^{42,45,46}$ In the following, we show that in the present case such disorder effects are weak and are unlikely to explain the observed anticrossing in the linewidths seen in Figure $5 \mathrm{c}$. We support this conclusion by direct, real-time measurements of the polariton dynamics.

Homogeneity of the J-Aggregates Resonance. Broader UP and narrower LP widths at zero detuning have also been observed in early reports on semiconductor microcavities ${ }^{42,45,46}$ and for J-aggregate molecules coupled to metallic nanovoids. ${ }^{19}$ These effects have mainly been explained by motional narrowing of polaritons $^{42-44}$ and other structural disorder effects, which are apparently much less pronounced in our system. ${ }^{45}$

To study possible disorder effects on the optical properties of our sample, we have performed spectral interferometry measurements on a thin J-aggregate layer deposited on a planar gold film. The reflectivity spectrum $R_{\mathrm{X}}$ and spectral phase $\varphi_{\mathrm{X}}$ of the coated gold 

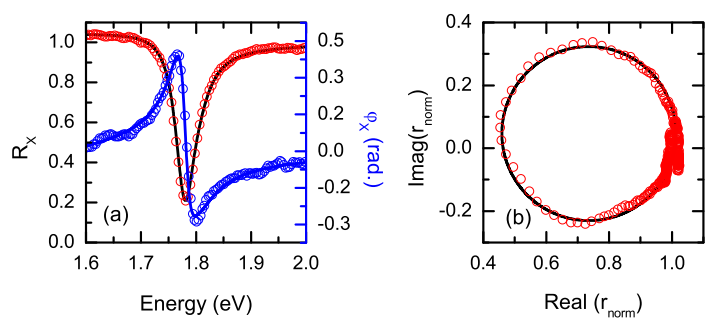

Figure 7. (a) Observed (red circles) and fitted (solid black) reflectivity spectra $R_{\mathrm{X}}(\omega)$, and corresponding observed (blue circles) and fitted (solid blue) spectral phase $\varphi(\omega)$, measured on a coated planar gold film at the incidence angle of $20^{\circ}$. (b) The corresponding observed normalized reflection coefficient $r_{\text {norm }}$ plotted in complex plane (red circles). The black circle is the fitted result to a circular function.

film are obtained at an incidence angle of $20^{\circ}$, as shown in Figure 7a.

To evaluate inhomogeneous broadening, we plot the spectral response in the complex plane, as shown in Figure $7 \mathrm{~b} .{ }^{47}$ In such a representation, the spectrum of a single, purely homogeneously broadened Lorentzian resonance is located on a nearly perfectly circular trace, provided that the susceptibility of the dye film is much less than unity. An inhomogeneously broadened system, however, would be represented by a more or less severely flattened, elliptical shape. We fit the complex reflectivity spectrum $\left(R_{\mathrm{X}}\right)^{1 / 2} \exp \left(i \varphi_{\mathrm{X}}\right)$ to the optical response of the hybrid system within the framework of the transfer matrix formalism, describing the permittivity of the dye layer as purely homogenously broadened Lorentzian resonances. Evidently, the experimental data closely follow a circular trace and show a very good match with the simulated data (solid line in Figure $7 \mathrm{~b}$ ). We conclude that the main resonance of the J-aggregate film is adequately described by a single Lorentzian line shape, suggesting that in the present samples inhomogeneous broadening is apparently weak.

Ultrafast Real-Time Dynamics of the Hybrid System. To provide independent experimental evidence for suband superradiance in the present hybrid system, we directly monitored the UP and LP population dynamics in time-resolved resonant pump-probe measurements. These experiments are performed at room temperature and under vacuum conditions using a home-built femtosecond pump-probe spectrometer, ${ }^{21,48}$ based on a noncollinear optical parametric amplifier. Broadband, $p$-polarized 15-fs-pulses centered at $1.8 \mathrm{eV}$ are used to resonantly excite the hybrid structure and to monitor the differential reflectivity, $\Delta R / R=\left(R_{\text {on }}-R_{\text {off }}\right) / R_{\text {off }}$ of the probe pulse as a function of delay time. Here, $R_{\text {on }}$ and $R_{\text {off }}$ denote the reflected probe spectrum in the presence and absence of the pump, respectively.

We first measured the population dynamics of uncoupled excitons. For this, we recorded $\Delta R / R$ spectra for a J-aggregate dye film deposited on a gold mirror without a grating, see Figure $8 \mathrm{a}$. Then, measurements
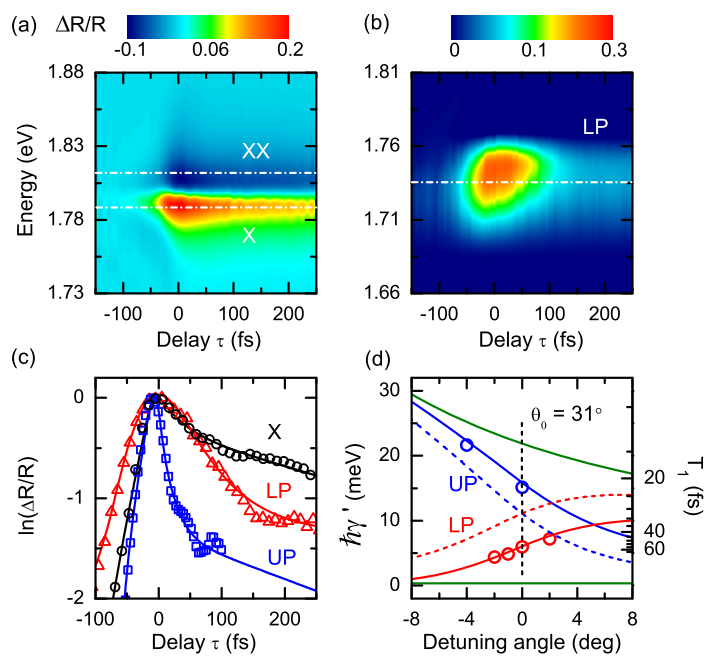

Figure 8. Time-resolved differential reflectivity signal $(\Delta R / R)$ (a) for a bare dye film and (b) for a hybrid structure at zero detuning $\theta_{0}$, measured for resonant excitation around $1.8 \mathrm{eV}$. The relevant resonances are marked (dashed lines). (c) $\Delta R / R$ dynamics (logarithmic scale) at the $\mathrm{X}$ resonance of the bare dye film, and near the LP $(1.74 \mathrm{eV})$ and UP $(1.84 \mathrm{eV})$ resonances of the hybrid structure at $\theta_{0}$. (d) Measured polariton population damping term $\hbar \gamma^{\prime}$ as a function of detuning angle $\theta-\theta_{0}$ (circles) compared to predictions of the coupled oscillator model (solid). Dashed lines denote $\hbar \gamma^{\prime}$ in the absence of incoherent photon exchange, $\gamma_{X P}=0$. Green: $\hbar \gamma^{\prime}$ of uncoupled $X$ and SPP modes.

were performed for coated groove arrays at $\theta_{0}$. Figure $8 \mathrm{~b}$ shows results obtained with spectrally narrowed pump pulses, predominantly exciting the LP resonance. Such measurements probe the population lifetime of the LP mode, in contrast to experiments with broader pump pulses, which excite a coherent superposition of UP and LP modes and show Rabi oscillations, reflecting the periodic energy transfer between Xs and SPPs. ${ }^{30}$

The bare dye data show a bleaching of the $X$ absorption around $1.78 \mathrm{eV}$ and an induced absorption peak at $1.82 \mathrm{eV}$ from one-exciton to two-exciton transitions. The dynamics of the $\Delta R / R$ signal at the $\mathrm{X}$ transition (Figure $8 \mathrm{a}$ ) reveals a perturbed free-induction decay of the $X$ polarization at $\tau<0$ and a biexponential decay ${ }^{49}$ with a fast decay time reflecting a weak coherence spike and a slower decay corresponding to the lifetime of the $X$ state of $\sim 600 \mathrm{fs}$. A fit to a biexponential decay convoluted with a Gaussian response function is shown as a solid line in Figure 8c.

Much faster decay dynamics are found in the hybrid system. For the LP mode, we find a rapid exponential decay of $\Delta R$ indicating an LP lifetime of only $55 \mathrm{fs}$. A weak signal remaining for $\tau>200 \mathrm{fs}$ likely results from probing a small fraction of uncoupled Xs. An even faster decay of the optical nonlinearity is seen when resonantly exciting the UP mode. Here, the $\Delta R$ signal decays to less than 0.1 of its maximum value with a decay time of only $\sim 22 \mathrm{fs}$. We have also measured polariton lifetimes at other detunings (Figure $8 \mathrm{~d}$ ), 
quantitatively confirming the predictions indicated as solid lines in Figure $8 \mathrm{~d}$. The data provide clear evidence for distinctly different population lifetimes of UP and LP modes at zero detuning. The observed lifetimes and their variation with detuning are well understood in terms of the coupled oscillator model, eq 1 . This suggests that in our system, the comparatively long LP lifetime at zero detuning reflects a subradiant damping of the polariton mode, resulting from the interplay between coherent dipole-dipole interaction and incoherent photon exchange. This convincing agreement of the measured lifetime with the predictions of the homogeneously broadened coupled oscillator model together with the observation of homogeneously broadened optical spectra suggests that structural disorder within the probed ensemble of Xs and SPPs has only a minor effect on the linear optical spectra of the hybrid structures. Upper polariton lifetimes, which are shorter than those of the lower polariton branch, have also been observed in earlier experiments on inorganic semiconductor microcavities. $^{50-52}$ In these systems, the faster UP lifetime results from a rapid scattering of polaritons from the UP branch to large in-plane wave vector LP states or higher exciton states. This is followed by polariton relaxation into small k-vector LP states by acoustic phonon emission and reabsorption. Since the phonon scattering rates scale with the exciton fraction of the LP wave function, this results in the well-known polariton relaxation bottleneck effect. ${ }^{53}$ Typically, these phononmediated relaxation processes proceed on a picosecond time scale, much slower than the polariton lifetimes of few tens of femtosecond that we observe in our system. This suggests that such phonon-mediated scattering processes are of minor importance for the polariton relaxation in strongly coupled J-aggregate-plasmon hybrid structures. This supports our conclusion that the observed anticrossing in the polariton widths seen in Figure $5 \mathrm{c}$ indeed reflects the formation of sub- and superradiant states in strongly coupled exciton-SPP systems.

\section{CONCLUSIONS}

In conclusion, we have investigated the optical response of a J-aggregate/metal hybrid nanostructure in the strong coupling regime by means of phaseresolved linear optical spectroscopy and ultrafast pump-probe spectroscopy. We provide experimental evidence, both in the spectral and temporal domain, for distinctly different damping rates for the two resulting hybrid polariton states, in particular at zero detuning. We argue that these different damping rates reflect the formation of sub- and superradiant polariton modes in our system. This argumentation is based on the following key observations: (i) All linear optical spectra are quantitatively described within a line shape model for a homogeneously broadened system. Apparently, at room temperature, disorder-induced inhomogeneous broadening effects are weak in our hybrid system. (ii) In a broad range of detunings, the polariton energies, linewidths and oscillator strengths are well understood within the framework of a phenomenological coupled oscillator model, provided that an incoherent exchange of photon energy between excitons and SPPs is taken into account. It is important that this model has only a very limited number of freely adjustable parameters, essentially the Rabi splitting, the incoherent cross-damping rate and the pure dephasing of the exciton oscillator. (iii) Directly measured polariton lifetimes at different detunings are reproduced by the same oscillator model without further parameter adjustment.

Taken together, this evidence strongly suggests that the optical properties of our hybrid system are largely governed by the interplay between the coherent dipole coupling between excitons and SPPs and the incoherent exchange of photon energy between both systems. Despite the very different nature of both elementary excitations, the coupling between Xs and SPPs apparently not only controls the energetics of the systems but also the dynamics of the polariton modes. We believe that this is an interesting observation since it suggests that the coupling results in an efficient decoupling of the subradiant mode from the environment.

Since $\gamma_{\mathrm{XP}}<\left(\gamma_{\mathrm{X}} \gamma_{\mathrm{P}}\right)^{1 / 2}\left(\hat{\boldsymbol{\mu}}_{\mathrm{X}} \cdot \hat{\boldsymbol{\mu}}_{\mathrm{P}}\right){ }^{3}$ this decoupling is most efficient, and a perfectly dark mode may arise, if both subsystems have equal radiative damping $\left(\gamma_{X}=\gamma_{P}\right)$ and if their dipole moments are aligned in parallel $\left(\hat{\boldsymbol{\mu}}_{\mathrm{x}} \|\right.$ $\hat{\mu}_{\mathrm{P}}$ ). Hence, the spontaneous emission dynamics of the hybrid system can be continuously controlled by adjusting the radiative damping of the individual subsystems and their dipolar coupling. This is of interest for tailoring quantum interference phenomena in active plasmonic devices ${ }^{17}$ and indicates new possibilities for nanoplasmonic circuitry and quantum information processing. This certainly warrants further detailed study of the optical properties of strongly coupled hybrid systems, specifically of the interplay between disorder, strong coupling, radiative damping and polariton localization. Advanced spectroscopic techniques such as ultrafast two-dimensional optical spectroscopy are likely to provide new insight into those phenomena.

\section{MATERIALS AND METHODS}

J-Aggregate/Metal Hybrid Nanostructures. The investigated Jaggregate/metal hybrid nanostructures are comprised of gold nanogroove arrays $\left(150 \times 150 \mu \mathrm{m}^{2}\right)$ with periods of 400-460 nm. These arrays are coated with a 50-nm-thick film made of the cyanine dye 2,2'-dimethyl-8-phenyl-5,6,5', 
6'-dibenzothiacarbocyanine chloride (Hayashibara Biochemicals Laboratories, Inc.) dissolved in polyvinyl alcohol (PVA), methanol and water. The optical density of such a film is typically $0.4 .^{21}$ The groove array period is chosen such that the first order polymer-metal plasmon mode is resonant with the J-aggregate exciton at $\sim 1.79 \mathrm{eV}^{13,21}$ The depth $(30 \mathrm{~nm})$ and width $(45 \mathrm{~nm})$ of the grating grooves is optimized to minimize SPP radiative damping. ${ }^{13,40}$

Angle-Resolved Spectral Interferometry. For high-resolution, angle-resolved spectral interferometry, ${ }^{13,35}$ as schematically depicted in Figure $3 \mathrm{~b}, p$-polarized broadband pulses $(1.6-2 \mathrm{eV})$ from an ultrafast white-light source (Fianium SC-450-4) with 80$\mathrm{MHz}$-repetition rate are split into sample and reference pulses in a balanced and chirp-compensated Mach-Zehnder interferometer. The complex sample reflection coefficient, $r(\omega)=$ $\left(R_{0}(\omega)\right)^{1 / 2} \mathrm{e}^{i \rho(\omega)}=\tilde{E}_{s}(\omega) / \tilde{E}_{\mathrm{R}}(\omega)$ is obtained from independently measured reflectivity spectra, $R_{0}(\omega)$, and from the spectral phase, $\varphi(\omega)$, encoded in the interference patterns $\mid \tilde{E}_{s}(\omega)+$ $\left.\tilde{E}_{\mathrm{R}}(\omega) \mathrm{e}^{-i \omega \tau}\right|^{2}=\left(1+|r(\omega)|^{2}\right)\left|\tilde{E}_{\mathrm{r}}(\omega)\right|^{2}+2\left|\tilde{E}_{\mathrm{r}}(\omega)\right|\left|\tilde{E}_{\mathrm{s}}(\omega)\right| \cos [\omega \tau+$ $\varphi(\omega)]$. Both are recorded as a function of the incidence angle, $\theta$. Here, $\tilde{E}_{\mathrm{s}}(\omega)$ and $\tilde{E}_{\mathrm{r}}(\omega)$ denote the electric field amplitude of the reflected sample and the reference beam at frequency $\omega$, respectively.

In the time domain, the corresponding electric field amplitudes can then be written as $E_{\mathrm{r}}(t)=(1 / 2 \pi) \int_{-\infty}^{+\infty} \tilde{E}_{\mathrm{r}}(\omega) \mathrm{e}^{-i \omega t} \mathrm{~d} \omega$ and $E_{\mathrm{s}}(t)=(1 / 2 \pi) \int_{-\infty}^{+\infty} \tilde{r}(\omega) \tilde{E}_{\mathrm{r}}(\omega) \mathrm{e}^{-i \omega t} \mathrm{~d} \omega=\int_{-\infty}^{t} \tilde{r}\left(t^{\prime}\right) \tilde{E}_{\mathrm{r}}\left(t-t^{\prime}\right) \mathrm{d} t^{\prime}$. The Fourier transform $r(t)=(1 / 2 \pi) \int_{-\infty}^{+\infty} \tilde{r}(\omega) \mathrm{e}^{-i \omega t} \mathrm{~d} \omega$, thus, gives the response of the system to a fictitious $\delta$-pulse excitation, $E_{\mathrm{r}}(t)=$ $\delta(t)$. A measurement of $\tilde{r}(\omega)$, therefore, allows us to deduce the time structure of the total electric field emitted by the sample in response to a $\delta$-pulse excitation.

As shown in Figures 4 and 7, the experimental spectra are convincingly described by a sum of Lorentzian response functions, eq 3. This allows us to extract the response of the two polariton modes as

$$
\tilde{r}_{\mathrm{p}}(\omega)=i \beta \sum_{j=\mathrm{UP}, \mathrm{LP}}\left[\frac{\left|\mu_{j}\right|^{2} \mathrm{e}^{-i \phi_{j}}}{\omega-\omega_{j}+i \gamma_{j}}+\frac{\left|\mu_{j}\right|^{2} \mathrm{e}^{i \phi_{j}}}{\omega+\omega_{j}+i \gamma_{j}}\right]
$$

simply by subtracting the response of all other contributions to the reflectivity. This extraction is possible since both amplitude and phase of the phase function have been measured experimentally. The time structure of the electric field emitted by the hybrid polariton modes is then given by Fourier transformation as

$$
\begin{aligned}
E_{\mathrm{p}}(t) & =\frac{1}{2 \pi} \int_{-\infty}^{+\infty} \tilde{r}_{\mathrm{p}}(\omega) \mathrm{e}^{-i \omega t} \mathrm{~d} \omega \\
& =\beta \cdot \Theta(t) \sum_{j=U P, L P}\left|\mu_{j}\right|^{2} \cos \left(\omega_{j} t+\phi_{j}\right) \mathrm{e}^{-\gamma_{j} t}
\end{aligned}
$$

Here, $\Theta(t)$ denotes the Heaviside step function. The deduced polariton field $E_{\mathrm{p}}(t)$ is plotted in Figure $4 \mathrm{c}, \mathrm{d}$.

Optical Response of the J-Aggregated Dye Film. For the characterization of the optical response of the J-aggregate dye film spincoated onto a planar gold film, we describe the susceptibility of the J-aggregates dye film as two Lorentzian line shapes presented as $\chi(\omega)=\sum_{k=1}^{2} A_{k}\left[\mathrm{e}^{-i \phi_{k}} /\left(\omega_{0, k}-\omega-i \gamma_{k}\right)+\mathrm{e}^{i \phi_{k}} /\left(\omega_{0, k}+\omega+\right.\right.$ $\left.\left.i \gamma_{k}\right)\right]$. The first oscillator represents the strong and narrow J-aggregated exciton resonance centered around $1.79 \mathrm{eV}$ and the other denotes the broad and weak monomer shoulder at around $2 \mathrm{eV}$. We then take the dielectric function of the dye film as $\varepsilon_{\mathrm{m}}=1+f \cdot \chi(\omega)+(1-f) \cdot \chi_{\mathrm{PVA}}$ with the filling factor $f=0.1$ depending on the concentration of J-aggregates in the solution and the susceptibility of PVA $\chi_{\mathrm{PVA}}=1.4$. With these parameters, we then simulate the experimentally measured normalized complex optical response $r_{\text {norm }}=r_{\text {dye }} / r_{\text {gold }}$ of this air/dye/gold layered system within the framework of transfer matrix formalism. Here, $r_{\text {dye }}$ is reflection coefficient of the entire layered system and $r_{\text {gold }}$ is the reflection coefficient measured on an uncoated gold film.

Optical Spectra of the Hybrid System. To simulate the optical spectra of the hybrid system, we need the energies and effective dipole moments of the coupled polariton resonance. These can be deduced from an analysis of the effective
Hamiltonian, eq 1. The complex polariton energies are given in eq 2. The normalized UP $(|+\rangle)$ and $L P(|-\rangle)$ wave functions can be written as $| \pm\rangle=a_{ \pm}|10\rangle+b_{ \pm}|01\rangle$, where the one-exciton/nophoton state is $|10\rangle$ and the no-exciton/one-photon state is $|01\rangle$. The coefficients are $a_{ \pm}=(\Delta \pm A) / B_{ \pm}$and $b_{ \pm}=2 D / B_{ \pm}$with $A=$ $\left(\Delta^{2}+4 C D\right)^{1 / 2}$ and $B_{ \pm}=\left(|\Delta \pm A|^{2}+4|D|^{2}\right)^{1 / 2}$. Here, $\Delta=\widetilde{\omega}_{\mathrm{X}}-\widetilde{\omega}_{\mathrm{p}}$ $C=\Omega_{\mathrm{R}}-i \gamma_{\mathrm{XP}}$ and $D=\Omega_{\mathrm{R}}^{*}-i \gamma_{\mathrm{XP}}$. The fractions $\left|C_{\mathrm{X}}\right|^{2}=|a|^{2} /\left(|a|^{2}+\right.$ $\left.|b|^{2}\right)$ and $\left|C_{P}\right|^{2}=|b|^{2} /\left(|a|^{2}+|b|^{2}\right)$ then give the probability of finding the system in the exciton and plasmon state, respectively.

In this model, effective dipole moments of the UP and LP oscillators are then given as $\boldsymbol{\mu}_{ \pm}=a_{ \pm}\left(N_{\mathrm{X}}\right)^{1 / 2} \boldsymbol{\mu}_{\mathrm{X}}+b_{ \pm}\left(N_{\mathrm{P}}\right)^{1 / 2} \boldsymbol{\mu}_{\mathrm{P}}$. Dipole moments of a single X/SPP mode $\boldsymbol{\mu}_{\mathrm{XP}}$ are deduced from their radiative lifetimes. ${ }^{54}$ Here we use $\mu_{\mathrm{X}}=100 D^{54}$ and the frequency-dependent $\mu_{\mathrm{P}}$ ranging from 8000 to $9100 \mathrm{D}$. We estimate an exciton density $N_{\mathrm{X}}$ of about $10^{24} \mathrm{~m}^{-3}$ and plasmon density $N_{\mathrm{p}}$ ranging from $0.8 \times 10^{19}$ to $1.5 \times 10^{19} \mathrm{~m}^{-3}{ }^{30}$ Finally, the angle-dependent spectra $R_{0}(\theta, \omega)$ and the corresponding spectral phases $\varphi(\theta, \omega)$ are calculated by using eq 3 .

Conflict of Interest: The authors declare no competing financial interest.

Acknowledgment. We thank the Deutsche Forschungsgemeinschaft (SPP 1391 and DFG-NSF Materials World Network), Fondazione Cariplo ("Engineering of optical nonlinearities in plasmonic metamaterials"), European Commission (FP-7 INFRASTRUCTURES-2008-1, "Laserlab Europe II", Contract No. 228334 and "CRONOS" Project, Grant Agreement No. 280879) and the Korea Foundation for International Cooperation of Science and Technology (Global Research Laboratory project, K20815000003) for financial support.

\section{REFERENCES AND NOTES}

1. DeVoe, R. G.; Brewer, R. G. Observation of Superradiant and Subradiant Spontaneous Emission of Two Trapped lons. Phys. Rev. Lett. 1996, 76, 2049-2052.

2. Hettich, C.; Schmitt, C.; Zitzmann, J.; Kühn, S.; Gerhardt, I.; Sandoghdar, V. Nanometer Resolution and Coherent Optical Dipole Coupling of Two Individual Molecules. Science 2002, 298, 385-389.

3. Akram, U.; Ficek, Z.; Swain, S. Decoherence and Coherent Population Transfer Between Two Coupled Systems. Phys. Rev. A 2000, 62, 013413.

4. Schmid, S. I.; Evers, J. Interplay of Vacuum-Mediated Interand Intra-Atomic Couplings in a Pair of Atoms. Phys. Rev. A 2010, 81, 063805

5. Dintinger, J.; Klein, S.; Bustos, F.; Barnes, W. L.; Ebbesen, T. W. Strong Coupling between Surface Plasmon-Polaritons and Organic Molecules in Subwavelength Hole Arrays. Phys. Rev. B 2005, 71, 035424.

6. Dahmen, C.; Schmidt, B.; von Plessen, G. Radiation Damping in Metal Nanoparticle Pairs. Nano Lett. 2007, 7, 318322.

7. Yoshie, T.; Scherer, A.; Hendrickson, J.; Khitrova, G.; Gibbs, H. M.; Rupper, G.; Ell, C.; Shchekin, O. B.; Deppe, D. G. Vacuum Rabi Splitting with a Single Quantum Dot in a Photonic Crystal nanocavity. Nature 2004, 432, 200-203.

8. Boca, A.; Miller, R.; Birnbaum, K. M.; Boozer, A. D.; McKeever, J.; Kimble, H. J. Observation of the Vacuum Rabi Spectrum for One Trapped Atom. Phys. Rev. Lett. 2004, 93, 233603.

9. Dicke, R. H. Coherence in Spontaneous Radiation Processes. Phys. Rev. 1954, 93, 99-110.

10. Lim, S.-H.; Bjorklund, T. G.; Spano, F. C.; Bardeen, C. J. Exciton Delocalization and Superradiance in Tetracene Thin Films and Nanoaggregates. Phys. Rev. Lett. 2004, 92, 107402.

11. Chen, Y. N.; Chuu, D. S.; Brandes, T. Current Detection of Superradiance and Induced Entanglement of Double Quantum Dot Excitons. Phys. Rev. Lett. 2003, 90, 166802.

12. Hübner, M.; Kuhl, J.; Stroucken, T.; Knorr, A.; Koch, S. W.; Hey, R.; Ploog, K. Collective Effects of Excitons in MultipleQuantum-Well Bragg and Anti-Bragg Structures. Phys. Rev. Lett. 1996, 76, 4199-4202. 
13. Ropers, C.; Park, D. J.; Stibenz, G.; Steinmeyer, G.; Kim, J.; Kim, D. S.; Lienau, C. Femtosecond Light Transmission and Subradiant Damping in Plasmonic Crystals. Phys. Rev. Lett. 2005, 94, 113901.

14. Wang, H.; Brandl, D. W.; Le, F.; Nordlander, P.; Halas, N. J. Nanorice: A Hybrid Plasmonic Nanostructure. Nano Lett. 2006, 6, 827-832

15. Sonnefraud, Y.; Verellen, N.; Sobhani, H.; Vandenbosch, G. A.; Moshchalkov, V. V.; Van Dorpe, P.; Nordlander, P.; Maier, S. A. Experimental Realization of Subradiant, Superradiant, and Fano Resonances in Ring/Disk Plasmonic Nanocavities. ACS Nano 2010, 4, 1664-1670.

16. Luk'yanchuk, B.; Zheludev, N. I.; Maier, S. A.; Halas, N. J.; Nordlander, P.; Giessen, H.; Chong, C. T. The Fano Resonance in Plasmonic Nanostructures and Metamaterials. Nat. Mater. 2010, 9, 707-715.

17. Martin-Cano, D.; Martin-Moreno, L.; Garcia-Vidal, F. J.; Moreno, E. Resonance Energy Transfer and Superradiance Mediated by Plasmonic Nanowaveguides. Nano Lett. 2010, 10, 3129-3134.

18. Bellessa, J.; Bonnand, C.; Plenet, J. C.; Mugnier, J. Strong Coupling between Surface Plasmons and Excitons in an Organic Semiconductor. Phys. Rev. Lett. 2004, 93, 036404.

19. Sugawara, Y.; Kelf, T. A.; Baumberg, J. J.; Abdelsalam, M. E.; Bartlett, P. N. Strong Coupling between Localized Plasmons and Organic Excitons in Metal Nanovoids. Phys. Rev. Lett. 2006, 97, 266808.

20. Vasa, P.; Pomraenke, R.; Schwieger, S.; Mazur, Y. I.; Kunets, V.; Srinivasan, P.; Johnson, E.; Kihm, J. E.; Kim, D. S.; Runge, E.; et al. Coherent Exciton/Surface-Plasmon-Polariton Interaction in Hybrid Metal-Semiconductor Nanostructures. Phys. Rev. Lett. 2008, 101, 116801.

21. Vasa, P.; Pomraenke, R.; Cirmi, G.; De Re, E.; Wang, W.; Schwieger, S.; Leipold, D.; Runge, E.; Cerullo, G.; Lienau, C. Ultrafast Manipulation of Strong Coupling in MetalMolecular Aggregate Hybrid Nanostructures. ACS Nano 2010, 4, 7559-7565.

22. Gomez, D. E.; Vernon, K. C.; Mulvaney, P.; Davis, T. J. Surface Plasmon Mediated Strong Exciton-Photon Coupling in Semiconductor Nanocrystals. Nano Lett. 2010, 10, 274278.

23. Schwartz, T.; Hutchison, J. A.; Genet, C.; Ebbesen, T. W. Reversible Switching of Ultrastrong Light-Molecule Coupling. Phys. Rev. Lett. 2011, 106, 196405.

24. Schlather, A. E.; Large, N.; Urban, A. S.; Nordlander, P.; Halas, N. J. Near-Field Mediated Plexcitonic Coupling and Giant Rabi Splitting in Individual Metallic Dimers. Nano Lett. 2013, 13, 3281-3286.

25. Pacifici, D.; Lezec, H. J.; Atwater, H. A. All-Optical Modulation by Plasmonic Excitation of CdSe Quantum Dots. Nat. Photonics 2007, 1, 402-406.

26. Pala, R. A.; Shimizu, K. T.; Melosh, N. A.; Brongersma, M. L. A Nonvolatile Plasmonic Switch Employing Photochromic Molecules. Nano Lett. 2008, 8, 1506-1510.

27. Noginov, M. A.; Zhu, G.; Mayy, M.; Ritzo, B. A.; Noginova, N.; Podolskiy, V. A. Stimulated Emission of Surface Plasmon Polaritons. Phys. Rev. Lett. 2008, 101, 226806.

28. Jaynes, E.; Cummings, F. W. Comparison of Quantum and Semiclassical Radiation Theories with Application to the Beam Maser. Proc. IEEE 1963, 51, 89-109.

29. Thompson, R. J.; Rempe, G.; Kimble, H. J. Observation of Normal-Mode Splitting for an Atom in an Optical Cavity. Phys. Rev. Lett. 1992, 68, 1132-1135.

30. Vasa, P.; Wang, W.; Pomraenke, R.; Lammers, M.; Maiuri, M.; Manzoni, C.; Cerullo, G.; Lienau, C. Real-time Observation of Ultrafast Rabi Oscillations between Excitons and Plasmons in J-aggregate/Metal hybrid Nanostructures. Nat. Photonics 2013, 7, 128-132.

31. Salomon, A.; Gordon, R. J.; Prior, Y.; Seideman, T.; Sukharev, M. Strong Coupling between Molecular Excited States and Surface Plasmon Modes of a Slit Array in a Thin Metal Film. Phys. Rev. Lett. 2012, 109, 073002.

32. Rempe, G.; Thompson, R.; Kimble, H. Cavity Quantum Electrodynamics with Strong-Coupling in the Optical Domain. Phys. Scr. 1994, T51, 67-77.
33. Lehmberg, R. H. Radiation from an N-Atom System. I. General Formalism. Phys. Rev. A 1970, 2, 883-888.

34. Milonnì, P. W.; Knight, P. L. Retarded Interaction of Two Nonidentical Atoms. Phys. Rev. A 1975, 11, 1090-1092.

35. Reynaud, F.; Salin, F.; Barthelemy, A. Measurement of Phase-Shifts Introduced by Nonlinear Optical Phenomena on Subpicosecond Pulses. Opt. Lett. 1989, 14, 275-277.

36. Weisbuch, C.; Nishioka, M.; Ishikawa, A.; Arakawa, Y. Observation of the Coupled Exciton-Photon Mode Splitting in a Semiconductor Quantum Microcavity. Phys. Rev. Lett. 1992, 69, 3314-3317.

37. Genet, C.; van Exter, M.; Woerdman, J. Fano-type Interpretation of Red Shifts and Red Tails in Hole Array Transmission Spectra. Opt. Commun. 2003, 225, 331-336.

38. Agarwal, G. S. Vacuum-Field Rabi Oscillations of Atoms in a Cavity. J. Opt. Soc. Am. B 1985, 2, 480-485.

39. Boyd, R. W. Nonlinear Optics, 2nd ed.; Academic Press: London, 2003.

40. Kim, D. S.; Hohng, S. C.; Malyarchuk, V.; Yoon, Y. C.; Ahn, Y.H.; Yee, K. J.; Park, J. W.; Kim, J.; Park, Q. H.; Lienau, C. Microscopic Origin of Surface-Plasmon Radiation in Plasmonic Band-Gap Nanostructures. Phys. Rev. Lett. 2003, 91, 143901.

41. Fidder, H.; Knoester, J.; Wiersma, D. A. Observation of the One-Exciton to Two-Exciton Transition in a J Aggregate. J. Chem. Phys. 1993, 98, 6564-6566.

42. Whittaker, D. M.; Kinsler, P.; Fisher, T. A.; Skolnick, M. S.; Armitage, A.; Afshar, A. M.; Sturge, M. D.; Roberts, J. S.; Hill, G. A. P. M. Motional Narrowing in Semiconductor Microcavities. Phys. Rev. Lett. 1996, 77, 4792-4795.

43. Whittaker, D. M. What Determines Inhomogeneous Linewidths in Semiconductor Microcavities? Phys. Rev. Lett. 1998, 80, 4791-4794.

44. Savona, V.; Piermarocchi, C.; Quattropani, A.; Tassone, F.; Schwendimann, P. Microscopic Theory of Motional Narrowing of Microcavity Polaritons in a Disordered Potential. Phys. Rev. Lett. 1997, 78, 4470-4473.

45. Ell, C.; Prineas, J.; Nelson, T. R.; Park, S.; Gibbs, H. M.; Khitrova, G.; Koch, S. W.; Houdré, R. Influence of Structural Disorder and Light Coupling on the Excitonic Response of Semiconductor Microcavities. Phys. Rev. Lett. 1998, 80, 4795-4798.

46. Laussy, F.P.; del Valle, E.; Tejedor, C. Strong Coupling of Quantum Dots in Microcavities. Phys. Rev. Lett. 2008, 101, 083601.

47. Jurna, M.; Garbacik, E. T.; Korterik, J. P.; Herek, J. L.; Otto, C.; Offerhaus, H. L. Visualizing Resonances in the Complex Plane with Vibrational Phase Contrast Coherent AntiStokes Raman Scattering. Anal. Chem. 2010, 82, 76567659.

48. Manzoni, C.; Polli, D.; Cerullo, G. Two-Color Pump-Probe System Broadly Tunable over the Visible and the Near Infrared with Sub-30 fs Temporal Resolution. Rev. Sci. Instrum. 2006, 77, 023103.

49. Guenther, T.; Lienau, C.; Elsaesser, T.; Glanemann, M.; Axt, V. M.; Kuhn, T.; Eshlaghi, S.; Wieck, A. D. Coherent Nonlinear Optical Response of Single Quantum Dots Studied by Ultrafast Near-Field Spectroscopy. Phys. Rev. Lett. 2002, 89, 057401.

50. Sermage, B.; Long, S.; Abram, I.; Marzin, J. Y.; Bloch, J.; Planel, R.; Thierry-Mieg, V. Time-Resolved Spontaneous Emission of Excitons in a Microcavity: Behavior of the Individual Exciton-Photon Mixed States. Phys. Rev. B 1996, 53, 16516-16523.

51. Bloch, J.; Marzin, J. Y. Photoluminescence Dynamics of Cavity Polaritons under Resonant Excitation in the Picosecond Range. Phys. Rev. B 1997, 56, 2103-2108.

52. Baumberg, J. J.; Armitage, A.; Skolnick, M. S.; Roberts, J. S. Suppressed Polariton Scattering in Semiconductor Microcavities. Phys. Rev. Lett. 1998, 81, 661-664.

53. Tassone, F.; Piermarocchi, C.; Savona, V.; Quattropani, A.; Schwendimann, P. Bottleneck Effects in the Relaxation and Photoluminescence of Microcavity Polaritons. Phys. Rev. B 1997, 56, 7554-7563.

54. Thränhardt, A.; Ell, C.; Khitrova, G.; Gibbs, H. M. Relation Between Dipole Moment and Radiative Lifetime in Interface Fluctuation Quantum Dots. Phys. Rev. B 2002, 65, 035327. 